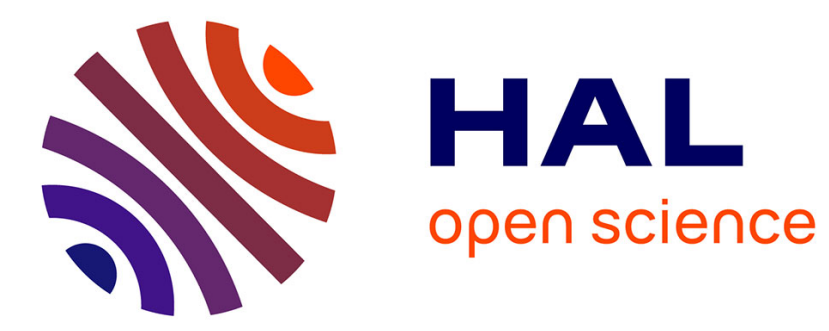

\title{
Integral ISS-Based Cascade Stabilization for Vectored-Thrust UAVs
}

Davide Invernizzi, Marco Lovera, Luca Zaccarian

\section{To cite this version:}

Davide Invernizzi, Marco Lovera, Luca Zaccarian. Integral ISS-Based Cascade Stabilization for Vectored-Thrust UAVs. IEEE Control Systems Letters, 2020, 4 (1), pp.43-48. 10.1109/LCSYS.2019.2921535 . hal-03029035

\section{HAL Id: hal-03029035 https://hal.science/hal-03029035}

Submitted on 27 Nov 2020

HAL is a multi-disciplinary open access archive for the deposit and dissemination of scientific research documents, whether they are published or not. The documents may come from teaching and research institutions in France or abroad, or from public or private research centers.
L'archive ouverte pluridisciplinaire HAL, est destinée au dépôt et à la diffusion de documents scientifiques de niveau recherche, publiés ou non, émanant des établissements d'enseignement et de recherche français ou étrangers, des laboratoires publics ou privés. 


\title{
Integral ISS-based cascade stabilization for vectored-thrust UAVs
}

\author{
Davide Invernizzi $^{1}$, Marco Lovera ${ }^{1}$ and Luca Zaccarian ${ }^{2}$
}

\begin{abstract}
We address stabilization of vectored-thrust Unmanned Aerial Vehicles (UAVs): a challenging task due to the peculiar nonlinear underactuated dynamics. According to a wellestablished approach based on the selection of suitable error variables, the error dynamics is described as a pseudo cascade connection where the attitude subsystem indirectly stabilizes the position dynamics. Unlike existing works, we address stability of this cascade using integral Input to State Stability (iISS). With this extension it is possible to employ a quasi time-optimal control law to achieve global asymptotic stabilization of any desired position, thus improving the transient performance with respect to existing control designs. A simulation example shows the performance improvement in comparison with a nested saturations stabilizer.
\end{abstract}

Index Terms-UAVs, integral ISS,

\section{INTRODUCTION}

V ECTORED-THRUST Unmanned Aerial Vehicles (UAVs) are platforms endowed with a propulsive system that can deliver a torque in any direction of the aircraft frame but a force only along a fixed axis: the thrust vector. Due to this underactuation, the position dynamics can be stabilized only through suitable attitude motions. There is a significant body of literature, well surveyed in [1], dealing with the stabilization problem for vectored-thrust UAVs as formulated in this paper, which is challenging also because it evolves on a nonlinear manifold corresponding to the set of rigid body motions. Early works on the subject date back to [2] and [3], while recent approaches are presented in [4], [5], [6].

The goal of this paper is to propose a control design which overcomes the limitations, in terms of transient performance, of modern strategies developed to globally stabilize the position of underactuated UAVs. In particular, the control designs proposed in [4], [5] as well as in our previous works [6], [7] ensure stability of the intrinsic cascade via a reduction approach [8] assessing the necessary property of "boundedness of solutions" by requiring the position stabilizer to possess Input to State Stabiliy (ISS) with respect to small inputs [9]. This small-signal ISS property is typically guaranteed by employing nested saturations-based stabilizers [5]: although very robust, this solution is known to yield poor transient performance [10].

Research supported in part by ANR via grant HANDY, number ANR-18CE40-0010.

${ }^{1}$ Davide Invernizzi and Marco Lovera are with Dipartimento di Scienze e Tecnologie Aerospaziali, Politecnico di Milano, Via La Masa 34, 20156 Milano, Italy \{davide.invernizzi, marco.lovera\}epolimi.it

${ }^{2}$ Luca Zaccarian is with CNRS, LAAS, 7 avenue du colonel Roche, F-31400 Toulouse, France, Univ. de Toulouse, LAAS, F-31400 Toulouse, France, and Dipartimento di Ingegneria Industriale, University of Trento, Italy. zaccarian@laas.fr
In this paper, we propose a hierarchical control design that extends the class of allowable position stabilizers to solutions having less stringent robustness requirements, thereby allowing for a more aggressive behavior. In particular, after introducing the UAV dynamics and the control problem in Section II, we borrow tools from stability analysis of integral ISS cascades [11], [12], and we propose in Section III a control architecture inducing an autonomous pseudo-cascade structure of the closed-loop error dynamics while giving freedom in the selection of the attitude and position stabilizers and in the shape of the interconnection term, as long as they satisfy certain properties always ensuring boundedness of solutions. Then in Section IV, we select these three basic components using high performance strategies. In particular, we suggest using an exponential attitude stabilizer [13] and a Quasi TimeOptimal (QTO) stabilizer [14] and we prove that this choice makes the UAV position dynamics integral ISS. The QTO stabilizer is the prototypical example of aggressive solutions, achieving excellent transient performance, which cannot be included in existing designs, notably [4], [5], [6], since proving (the previously required) small-signal ISS property is hard if not impossible. A simulation example discussed in Section V highlights the advantage of the proposed solution with respect to a nested saturations approach in a scenario characterized by a significant initial configuration error.

Notation. $\mathbb{R}\left(\mathbb{R}_{>0}, \mathbb{R}_{\geq 0}\right)$ denotes the set of (positive, nonnegative) real numbers, $\mathbb{R}^{n}$ denotes the $n$-dimensional Euclidean space and $\mathbb{R}^{m \times n}$ the set of $m \times n$ real matrices. The canonical basis in $\mathbb{R}^{n}$ is denoted as $e_{i}:=(0, \ldots, 1, \ldots, 0)$ for $i \in\{1, \ldots, n\}$ and the identity matrix in $\mathbb{R}^{n \times n}$ is denoted as $I_{n}:=\left[e_{1} \cdots e_{i} \cdots e_{n}\right]$. Given $x=\left(x_{1}, \ldots, x_{n}\right) \in \mathbb{R}^{n},\|x\|:=\sqrt{x_{1}^{2}+\ldots+x_{n}^{2}}$ is the Euclidean norm while for a matrix $A \in \mathbb{R}^{n \times n},\|A\|_{F}:=\sqrt{\operatorname{tr}\left(A^{T} A\right)}$ is the Frobenius norm and $\operatorname{skew}(A):=\frac{A-A^{T}}{2}$ is the skew-symmetric part of $A$. The set $\mathrm{SO}(3):=\left\{R \in \mathbb{R}^{3 \times 3}: R^{T} R=I_{3}, \operatorname{det}(R)=1\right\}$ denotes the thirdorder Special Orthogonal group while $\mathbb{S}^{n}:=\left\{q \in \mathbb{R}^{n+1}:\|q\|=1\right\}$ denotes the $n$-dimensional unit sphere. where the normalized distance with respect to $I_{3}$, induced by the Frobenius norm, is denoted as $\|R\|_{\mathrm{SO}(3)}:=\frac{1}{8}\left\|R-I_{3}\right\|_{F}=\sqrt{\frac{1}{4} \operatorname{tr}\left(I_{3}-R\right)} \in[0,1]$. Given $\omega \in \mathbb{R}^{3}$, the hat map $\hat{\imath}: \mathbb{R}^{3} \rightarrow \mathfrak{s o}(3):=\left\{\Omega \in \mathbb{R}^{3 \times 3}: \Omega=-\Omega^{T}\right\}$ is such that $\hat{\omega} y=$ $\omega \times y, \forall y \in \mathbb{R}^{3}$ and $\times$ represents the cross product in $\mathbb{R}^{3}$. The inverse of the hat map is the vee map, denoted as $(\cdot)^{\vee}: \mathfrak{s o}(3) \rightarrow \mathbb{R}^{3}$. We use standard comparison functions from [15]: function $\alpha: \mathbb{R}_{\geq 0} \rightarrow \mathbb{R}_{\geq 0}$ is of class $K$ if it is zero at zero, strictly increasing, and continuous. It is of class $K_{\infty}$ if it is also unbounded. $\beta: \mathbb{R}_{\geq 0} \times \mathbb{R}_{\geq 0} \rightarrow \mathbb{R}_{\geq 0}$ is of class $K L$ if it is of class $K$ in the first argument and nonincreasing and converging to zero as its second argument tends to $+\infty$. 


\section{DYNAMICAL MODEL AND CONTROL PROBLEM}

The configuration of a UAV is globally and uniquely described by the pair $(R, x) \in \mathrm{SO}(3) \times \mathbb{R}^{3}$, where $R$ and $x$ are, respectively, the rotation matrix and the position vector of a body-fixed frame $\mathscr{F}_{B}=\left(O_{B},\left\{b_{1}, b_{2}, b_{3}\right\}\right)$ with respect to an inertial frame $\mathscr{F}_{I}=\left(O_{I},\left\{i_{1}, i_{2}, i_{3}\right\}\right)$. A reasonable model for control design is described by the following set of differential equations [1]:

$$
\begin{array}{ll}
\dot{x}=v & m \dot{v}=-m g e_{3}+R f_{c} \\
\dot{R}=R \hat{\omega} & J \dot{\omega}=-\omega \times J \omega=-\hat{\omega} J \omega+\tau_{c},
\end{array}
$$

where $\omega \in \mathbb{R}^{3}$ is the body angular velocity, $v \in \mathbb{R}^{3}$ is the translational velocity of the center of mass, resolved in $\mathscr{F}_{I}$, $m \in \mathbb{R}_{>0}$ and $J=J^{T} \in \mathbb{R}_{>0}^{3 \times 3}$ are the mass and the body inertia matrix of the UAV, respectively, and $g=9.81 \mathrm{~m} / \mathrm{s}^{2}$ represents the gravitational acceleration. Finally, $f_{c} \in \mathbb{R}^{3}$ is the control force and $\tau_{c} \in \mathbb{R}^{3}$ the control torque, both resolved in $\mathscr{F}_{B}$. The propulsive system of vectored-thrust UAVs allows to produce a (bounded) control force directed only along the positive direction of $b_{3}$, i.e., the components of $f_{c}$ in the body frame must satisfy

$$
f_{c}=T_{c} e_{3}, \quad 0<T_{c} \leq T_{M} \in \mathbb{R}_{>0},
$$

where $T_{c}$ is the overall thrust that can be assigned by properly modulating the thrust delivered by each propeller. It is assumed that the control torque $\tau_{c}$ spans $\mathbb{R}^{3}$, i.e., that the rotational dynamics (2) is fully actuated.

In this paper we exploit a cascade-based design for the attitude dynamics and consider $\omega_{c}:=\omega$ as a control input, under the assumption that the attitude dynamics in equation (2) can be made sufficiently fast in tracking $\omega_{c}$ by a suitable selection of $\tau_{c}$ (for a discussion about this approximation, see [1]). In this case, the model for control design reduces to:

$$
\begin{aligned}
\dot{x} & =v \\
m \dot{v} & =-m_{g} e_{3}+T_{c} R e_{3} \\
\dot{R} & =R \hat{\omega}_{c}
\end{aligned}
$$

where the attitude motion (6) is independent of the translational one (4),(5). This structure is exploited in the hierarchical control strategy presented in the next section.

This work focuses on the stabilization of the underactuated model (4)-(6). According to this model, it is either possible to stabilize a desired attitude and altitude or a desired position and rotation about the gravity axis $e_{3}$. This paper addresses the latter problem under the following standard assumption.

Assumption 1: 1) the desired trajectory is a constant reference $\left(x_{d}, 0, R_{d}\right) \in \mathbb{R}^{3} \times \mathbb{R}^{3} \times \mathrm{SO}(3)$ such that $\left.R_{d} e_{3}=e_{3} ; 2\right)$ the maximum available thrust $T_{M}$ is larger than the UAV weight, i.e., $\left.T_{M}>m g ; 3\right)$ the state of the system $(x, v, R)$ is available for feedback.

While the first part of the assumption is necessary to account for the platform underactuation when constraint (3) is included in the dynamical model, the second part is necessary control authority requirement to have a solvable control problem. In view of Assumption 1, the control problem can be formally stated as follows.
Problem 1: Consider the dynamical model described by equations (4)-(6) and assume that the control thrust $T_{C}$ is bounded as $0<T_{c} \leq T_{M}$ for a strictly positive scalar $T_{M}$. Find a state feedback control law for $u:=\left(T_{c}, \omega_{c}\right) \in\left(0, T_{M}\right] \times \mathbb{R}^{3}$ such that any constant set point $\left(x_{d}, 0, R_{d}\right) \in \mathbb{R}^{3} \times \mathbb{R}^{3} \times \mathrm{SO}(3)$ satisfying Assumption 1, is asymptotically stable.

\section{CONTROL DESIGN AND STABILITY ANALYSIS}

This section is devoted to presenting the control design and the corresponding stability analysis. In [4], [5], [6] a smallsignal ISS property was the basic requirement for the position stabilizer to ensure stability of the closed-loop system. Here we relax this requirement and enlarge the class of allowable position stabilizers towards solutions capable of superior performance. To this end, we invoke recent results on stabilization of nonlinear cascades, based on integral ISS arguments (see equation (24) below, and [11] for additional details about iISS). The formulation that we propose leaves the door open for different selections of stabilizers of the nominal position and attitude dynamics, therefore parametrizing a family of solutions to Problem 1.

\section{A. Control law and closed-loop dynamics}

We propose an attitude planner paradigm, similar to the one presented in our previous work [6], to tackle the system underactuation. Let us first introduce the stabilization error

$$
e_{x}:=x-x_{d}, \quad e_{v}:=v, \quad R_{e}:=R_{p}^{T}\left(e_{x}, e_{v}, R_{d}\right) R,
$$

where $R_{p}: \mathbb{R}^{3} \times \mathbb{R}^{3} \times \mathrm{SO}(3) \rightarrow \mathrm{SO}(3)$ is the reference attitude provided by the attitude planner (see Figure 1) defined as:

$$
R_{p}\left(e_{x}, e_{v}, R_{d}\right):=\left[\frac{b_{p_{3}} \times R_{d} e_{1}}{\left\|b_{p_{3}} \times R_{d} e_{1}\right\|} \times b_{p_{3}} \quad \frac{b_{p_{3}} \times R_{d} e_{1}}{\left\|b_{p_{3}} \times R_{d} e_{1}\right\|} \quad b_{p_{3}}\right],
$$

where $b_{p_{3}}:=\frac{f_{d}\left(e_{x}, e_{v}\right)}{\left\|f_{d}\left(e_{x}, e_{v}\right)\right\|}$ and

$$
f_{d}\left(e_{x}, e_{v}\right):=\gamma_{p}\left(e_{x}, e_{v}\right)+m g e_{3}
$$

is guaranteed to never vanish by design. Based on (8)-(9) we select the inputs of dynamics (4)-(6) as

$$
\begin{aligned}
T_{c} & =c\left(R_{e}\right) e_{3}^{T} R_{p}^{T}\left(e_{x}, e_{v}, R_{d}\right) f_{d}\left(e_{x}, e_{v}\right) \\
\omega_{c} & =\gamma_{R}\left(R_{e}\right)+R_{e}^{T} \omega_{p}\left(e_{x}, e_{v}, R_{d}\right),
\end{aligned}
$$

where $\gamma_{p}: \mathbb{R}^{3} \times \mathbb{R}^{3} \rightarrow \mathbb{R}^{3}$ and $\gamma_{R}: \mathrm{SO}(3) \rightarrow \mathbb{R}^{3}$ are static state feedback stabilizers, $c(\cdot)$ is an interconnection term, to be selected, and $\omega_{p}\left(e_{x}, e_{v}, R_{d}\right):=\left(R_{p}^{T} \dot{R}_{p}\right)^{\vee}$ is the angular velocity reference computed by the attitude planner (see [6], [7] for details).

Proposition 1: Consider dynamics (4)-(6) with the stabilization errors in (7) and controller (10)-(11). If $f_{d}\left(e_{x}, e_{v}\right) \neq 0$ $\forall\left(e_{x}, e_{v}\right) \in \mathbb{R}^{6}$, then the closed-loop dynamics reads:

$$
\begin{aligned}
\dot{e}_{x} & =e_{v} \\
m \dot{e}_{v} & =\gamma_{p}\left(e_{x}, e_{v}\right)+\Delta R\left(R_{e}, e_{x}, e_{v}, R_{d}\right) f_{d}\left(e_{x}, e_{v}\right) \\
\dot{R}_{e} & =R_{e} \hat{\gamma}_{R}\left(R_{e}\right)
\end{aligned}
$$

where

$$
\Delta R\left(R_{e}, e_{x}, e_{v}, R_{d}\right):=c\left(R_{e}\right) R_{p}\left(e_{x}, e_{v}, R_{d}\right) R_{e} R_{p}^{T}\left(e_{x}, e_{v}, R_{d}\right)-I_{3}
$$




$$
\dot{R}_{e}=R_{e} \hat{\gamma}_{R}\left(R_{e}\right) \stackrel{R_{e}}{\longrightarrow} \begin{gathered}
\dot{e}_{x}=e_{v} \\
m \dot{e}_{v}=\gamma_{p}\left(e_{x}, e_{v}\right)+\Delta R\left(R_{e}, e_{x}, e_{v}, R_{d}\right) f_{d}\left(e_{x}, e_{v}\right)
\end{gathered}
$$

Figure 1. Cascade structure of the error dynamics (equations (12)-(14)).

Proof. We start by inspecting the position dynamics in (4), (5) and refer to the position and velocity errors defined in (7). By taking their time derivative along (4)-(5), we get

$$
\begin{aligned}
\dot{e}_{x} & =e_{v} \\
m \dot{e}_{v} & =m\left(\dot{v}-\dot{v}_{d}\right)=-m g e_{3}+T_{c} R e_{3} .
\end{aligned}
$$

By noting that $T_{c} e_{3}=c\left(R_{e}\right) R_{p}^{T} f_{d}$, equation (17) becomes

$$
m \dot{e}_{v}=-m g e_{3}+c\left(R_{e}\right) R R_{p}^{T} f_{d} .
$$

The velocity error dynamics can be further rewritten as

$$
\begin{aligned}
m \dot{e}_{v} & =-m g e_{3}+c\left(R_{e}\right) R_{p} R_{p}^{T} R R_{p}^{T} f_{d} \\
& =-m g e_{3}+c\left(R_{e}\right) R_{p} R_{e} R_{p}^{T} f_{d} .
\end{aligned}
$$

Finally, adding and subtracting $f_{d}$ from equation (9), we get

$$
m \dot{e}_{v}=\gamma_{p}\left(e_{x}, e_{v}\right)+\left(c\left(R_{e}\right) R_{p} R_{e} R_{p}^{T}-I_{3}\right) f_{d},
$$

corresponding to $\Delta R\left(R_{e}, e_{x}, e_{v}, R_{d}\right)$ in (15). For the attitude dynamics, using the definition of $R_{e}$ in (7), we get:

$$
\begin{aligned}
\dot{R}_{e} & =\dot{R}_{p}^{T} R+R_{p} \dot{R}=-\hat{\omega}_{p} R_{p}^{T} R+R_{p}^{T} R \hat{\omega} \\
& =R_{p}^{T} R\left(\hat{\omega}_{c}-\left(R_{p} R\right)^{T} \hat{\omega}_{p} R_{p}^{T} R\right)=R_{e}\left(\hat{\omega}_{c}-R_{e}^{T} \hat{\omega}_{p} R_{e}\right) .
\end{aligned}
$$

Finally, using controller (11) and using property $\left(R_{e} \omega_{p}\right)^{\wedge}=R_{e}^{T} \hat{\omega}_{p} R_{e}$, the closed-loop (12)-(14) is obtained.

Remark 1: The position error (12)-(13) is affected by the attitude error through the term $\Delta R f_{d}$, which weighs the mismatch between the desired force $f_{d}$ in (9) and the control force resolved in $\mathscr{F}_{I}$, i.e., $R f_{c}$. As shown in [6], the interconnection function $c(\cdot)$ in $(15)$ helps improving the transient performance by shaping the mismatch term.

\section{B. Cascade stability analysis and stabilizers}

Control law (10)-(11) induces a pseudo cascade structure of the closed-loop dynamics (Figure 1) while leaving freedom in the selection of the attitude and position stabilizers $\gamma_{R}(\cdot)$ and $\gamma_{p}(\cdot, \cdot)$, as well as in the selection of the connection term $\Delta R$, which depends upon the scaling function $c(\cdot)$. Clearly, their selection must guarantee asymptotic stabilization of the equilibrium $\left(R_{e}, e_{x}, e_{v}\right)=\left(I_{3}, 0,0\right)$. The following definition [12] will be used to characterize the admissible iISS gains when the speed of convergence of the perturbing subsystem is exponential.

Definition 1: A function $\eta(\cdot)$ is said to be of class- $H_{I}$ if it is of class- $K$ and satisfies $\int_{0}^{1} \frac{\eta(s)}{s} d s<\infty$.

The following result is a slightly modified version of [12, Corollary 2], adapted to our system evolving on $\mathbb{R}^{6} \times \mathrm{SO}(3)$.

Lemma 1: Let $x \in \mathbb{R}^{6}$ and $R \in \mathrm{SO}(3)$ and consider the cascade $\dot{x}=f(x, R), \dot{R}=Q(R)$ where $f: \mathbb{R}^{6} \times \mathrm{SO}(3) \rightarrow \mathbb{R}^{6}$ and $Q: \mathrm{SO}(3) \rightarrow T \mathrm{SO}(3)$ are smooth vector fields with $f\left(0, I_{3}\right)=0$ and $Q\left(I_{3}\right)=0$. Suppose that the equilibrium $R=I_{3}$ is locally exponentially stable (LES) for $\dot{R}=Q(R)$, namely there exist $c_{R}, \lambda_{R} \in \mathbb{R}_{>0}$ and $\ell \in(0,1)$ such that $\|R(0)\|_{\mathrm{SO}(3)} \leq \ell$ implies

$$
\|R(t)\|_{\mathrm{SO}(3)} \leq c_{R}\|R(0)\|_{\mathrm{SO}(3)} \exp \left(-\lambda_{R} t\right), t \geq 0 .
$$

If the dynamics $\dot{x}=f(x, R)$ is iISS with respect to input $R$ with a class- $H_{I}$ gain, namely, if there exist a class- $K_{\infty}$ function $\alpha(\cdot)$, a class- $K L$ function $\beta(\cdot)$, and a class- $H_{I}$ gain $\eta(\cdot)$ such that, for all $t \geq 0$,

$$
\alpha(\|x(t)\|) \leq \beta(\|x(0)\|, t)+\int_{0}^{t} \eta\left(\|R(\tau)\|_{\mathrm{SO}(3)}\right) d \tau,
$$

then $(x, R)=\left(0, I_{3}\right)$ is locally asymptotically stable with domain of attraction including the set $\left\{(x, R): x \in \mathbb{R}^{6}, R \in \mathrm{SO}(3),\|R\|_{\mathrm{SO}(3)} \leq \ell\right\}$.

Proof. The proof hinges upon the iISS property guaranteed by inequality (24). In particular, from (23) we have that $\|R(0)\|_{\mathrm{SO}(3)} \leq \ell$ implies

$$
\begin{aligned}
\int_{0}^{\infty} \eta\left(\|R(\tau)\|_{\mathrm{SO}(3)}\right) d \tau & \leq \int_{0}^{\infty} \eta\left(c_{R}\|R(0)\|_{\mathrm{SO}(3)} \exp \left(-\lambda_{R} \tau\right)\right) d \tau \\
& =\frac{1}{\lambda_{R}} \int_{0}^{c_{R}\|R(0)\|_{\mathrm{SO}(3)}} \frac{\eta(s)}{s} d s
\end{aligned}
$$

where we used $s:=c_{R}\|R(0)\|_{\mathrm{SO}(3)} \exp \left(-\lambda_{R} \tau\right)$. Then, because $\eta(\cdot)$ is of class- $H_{I}$, the function

$$
v(\bar{s}):=\frac{1}{\lambda_{R}} \int_{0}^{\bar{s}} \frac{\eta(s)}{s} d s
$$

is well-defined for all $\bar{s} \in \mathbb{R}_{\geq 0}$ and it is of class- $K$ because $v(0)=0$ and $\frac{\eta(s)}{s}>0$ for all $s>0$. Thus, from property (24), we can write:

$$
\alpha(\|x(t)\|) \leq \beta(\|x(0)\|, t)+v\left(c_{R}\|R(0)\|_{\mathrm{SO}(3)}\right) .
$$

This proves that all solutions are bounded. Furthermore, since $\int_{0}^{\infty} \eta\left(\|R(\tau)\|_{\mathrm{SO}(3)}\right) d \tau$ is bounded, (24) implies that $x(t) \rightarrow 0$ as $t \rightarrow \infty$ following the arguments in [11, Prop. 6].

System (12)-(14) can be written as in Lemma 1 by defining $e_{p}:=\left(e_{x}, e_{v}\right) \in \mathbb{R}^{6}$ and $R:=R_{e} \in \mathrm{SO}(3)$. Therefore, the stabilization of the cascade (12)-(14) is possible if one can provide a suitable attitude stabilizer $\gamma_{R}(\cdot)$ in (14) guaranteeing (local) exponential stability ${ }^{1}$ of the equilibrium $R_{e}=I_{3}$, a position stabilizer $\gamma_{p}(\cdot, \cdot)$ and a scaling function $c(\cdot)$ for which the position error dynamics (12)-(13) is iISS with respect to input $R_{e}$ in the sense of (24). In this spirit, we define the following properties.

Property 1: The attitude stabilizer $R_{e} \mapsto \gamma_{R}\left(R_{e}\right)$ is continuous and such that the equilibrium point $R_{e}=I_{3}$ is locally exponentially stable for (6) with domain of attraction containing $\left\{R \in \mathrm{SO}(3):\|R\|_{\mathrm{SO}(3)} \leq \ell\right\}$ for some $\ell \in(0,1)$.

Property 2: The position stabilizer $\left(e_{x}, e_{v}\right) \mapsto \gamma_{p}\left(e_{x}, e_{v}\right)$ is continuously differentiable and the scaling function $R_{e} \mapsto$ $c\left(R_{e}\right)$ is continuous and they are such that

\footnotetext{
${ }^{1}$ Due to topological obstructions of $\mathrm{SO}(3)$, only a local exponential result can be achieved with continuous static state feedback [16]. Nonetheless, it can be shown that the basin of attraction can be extended up to the set $\left\{R \in \mathrm{SO}(3):\|R\|_{\mathrm{SO}(3)}<1\right\}$ which does not contain only rotations about any axis of $\pi$ rad away from $I_{3}$. Moreover, the results can be extended to global ones by using hybrid stabilizers [17].
} 
1) there exist class- $K_{\infty}$ and $K L$ functions $\alpha(\cdot)$ and $\beta(\cdot)$ and a class- $H_{I}$ gain $\eta(\cdot)$ such that the $\left(e_{x}, e_{v}\right)$-subsystem in equations (12), (13), (15) is iISS from $R_{e}$ as in (24);

2) there exist saturation levels $M_{i} \in \mathbb{R}_{>0}, i \in\{1,2,3\}$, satisfying

$$
M_{3}<m g, \quad \sum_{i=1}^{3} M_{i} \leq T_{M}-m g
$$

and such that the components of $\gamma_{p}$ are bounded as $\left|\gamma_{p_{i}}\left(e_{x}, e_{v}\right)\right| \leq M_{i} \forall\left(e_{x}, e_{v}, i\right) \in \mathbb{R}^{6} \times\{1,2,3\}$.

Remark 2: The second item in Property 2 and the continuous differentiability of $\gamma_{p}(\cdot, \cdot)$ are required in order to obtain a planner reference $R_{p}\left(e_{x}, e_{v}, R_{d}\right)$ as defined in equations (8)-(9) that is well-defined and $C^{1} \forall\left(e_{x}, e_{v}, R_{d}\right) \in \mathbb{R}^{3} \times \mathbb{R}^{3} \times \mathrm{SO}(3)$ (see [6, Section IV-C] for additional details).

Based on the above properties and Lemma 1, the main result of the paper is then given by the following theorem.

Theorem 1: Consider the closed-loop system described by (4)-(6) controlled by (8)-(11). If $\gamma_{R}(\cdot), \gamma_{p}(\cdot, \cdot)$ and $c(\cdot)$ are selected according to Properties 1 and 2, then for any desired constant trajectory $\left(x_{d}, 0, R_{d}\right) \in \mathbb{R}^{3} \times \mathbb{R}^{3} \times \mathrm{SO}(3)$ satisfying Assumption 1, the control law (10)-(11) solves Problem 1, in particular the point $\left(e_{x}, e_{v}, R_{e}\right)=\left(0,0, I_{3}\right)$ is asymptotically stable with domain of attraction containing $\mathbb{R}^{3} \times \mathbb{R}^{3} \times$ $\left\{R \in \mathrm{SO}(3):\|R\|_{\mathrm{SO}(3)} \leq \ell\right\}$.

Proof. The proof follows the same steps as the proof of Lemma 1. Indeed, since the planner reference is $C^{1}$ and well-defined (see Remark 2), the control input $\omega_{c}$ in (11) is well-defined as well, and Proposition 1 ensures that the steps in Lemma 1 can be completed. Moreover, thanks to (28) and Assumption 1, the control force is bounded by $T_{M}$. $\square$

\section{SAMPle Stabilizers Design}

In this section we present a selection of the basic components of the control law, namely $\gamma_{p}(\cdot, \cdot), \gamma_{R}(\cdot), c(\cdot)$ and show that they satisfy Properties 1 and 2 , thereby guaranteeing the applicability of Theorem 1. Specifically, we adopt a quasi time-optimal strategy for position stabilization, adapted from [14], which consists of a locally Lipschitz state feedback that behaves linearly in a neighborhood of the origin, but coincides with the time-optimal bang-bang feedback in the presence of large velocity errors. The QTO stabilizer is a representative candidate of aggressive solutions with excellent transient performance, but limited robustness properties: while we are not able to prove that the QTO enjoys a small-signal ISS property, which makes it unfit for existing strategies, we show that it enjoys integral ISS as in (24).

\section{A. Attitude stabilization}

The next proposition gives an example of an attitude stabilizer for which Property 1 is satisfied.

Proposition 2: Given $\gamma_{R}\left(R_{e}\right):=-\frac{k_{R}}{\sqrt{1+\operatorname{tr}\left(R_{e}\right)}} \operatorname{skew}\left(R_{e}\right)^{\vee}$, where $k_{R} \in \mathbb{R}_{>0}$ is a scalar gain, Property 1 is satisfied for any $\ell \in(0,1)$. In particular, the (closed-loop) trajectories of (14) converge exponentially to $R_{e}=I_{3}$ for all initial conditions starting in set $S_{R}:=\left\{R \in \mathrm{SO}(3):\|R\|_{\mathrm{SO}(3)}<1\right\}$.
Proof. The proof follows from Lyapunov arguments, by considering the candidate Lyapunov function $V_{R}\left(R_{e}\right):=$ $k_{R}\left(2-\sqrt{1+\operatorname{tr}\left(R_{e}\right)}\right)$ which is quadratic in the error, namely, $k_{R}\left\|R_{e}\right\|_{\mathrm{SO}(3)}^{2} \leq V_{R}\left(R_{e}\right) \leq 2 k_{R}\left\|R_{e}\right\|_{\mathrm{SO}(3)}^{2} \quad$ (see [13, equation 12.4]), and continuously differentiable in $S_{R}$. Indeed, by taking its time derivative along the dynamics (14) and by exploiting the trace operator property $\operatorname{tr}(A \hat{y})=\operatorname{tr}(\operatorname{skew}(A) \hat{y})=$ $-2\left(\operatorname{skew}(A)^{\vee}\right)^{T} y, A \in \mathbb{R}^{3 \times 3}, y \in \mathbb{R}^{3}$, we get:

$$
\begin{aligned}
\dot{V}_{R}\left(R_{e}\right) & \left.=-\frac{k_{R}}{2 \sqrt{1+\operatorname{tr}\left(R_{e}\right)}} \operatorname{tr}\left(\dot{R}_{e}\right)\right) \underset{(14)}{=}-\frac{k_{R}}{2 \sqrt{1+\operatorname{tr}\left(R_{e}\right)}} \operatorname{tr}\left(R_{e} \hat{\gamma}_{R}\left(R_{e}\right)\right) \\
& =-\frac{k_{R}}{2 \sqrt{1+\operatorname{tr}\left(R_{e}\right)}} \operatorname{tr}\left(\operatorname{skew}\left(R_{e}\right) \hat{\gamma}_{R}\left(R_{e}\right)\right) \\
& =\frac{k_{R}\left(\operatorname{skew}\left(R_{e}\right)^{\vee}\right)^{T}}{\sqrt{1+\operatorname{tr}\left(R_{e}\right)}} \gamma_{R}\left(R_{e}\right)=-\left\|\gamma_{R}\left(R_{e}\right)\right\|^{2}
\end{aligned}
$$

which is continuous and negative definite in $S_{R}$ (note that $\gamma_{R}\left(R_{e}\right)$ is well-defined in $S_{R}$ since $\operatorname{tr}(R)=-1 \Leftrightarrow\|R\|_{\mathrm{SO}(3)}=$ 1). Furthermore, since it can be shown that $\left\|\gamma_{R}\left(R_{e}\right)\right\|=$ $k_{R}\left\|R_{e}\right\|_{\mathrm{SO}(3)} \forall R_{e} \in S_{R}$, equation (29) can be written as

$$
\dot{V}_{R}\left(R_{e}\right)=-k_{R}^{2}\left\|R_{e}\right\|_{\mathrm{SO}(3)}^{2} \leq-\frac{k_{R}}{2} V_{R}\left(R_{e}\right) \forall R_{e} \in S_{R} .
$$

If we take $R_{e}(0) \in S_{V_{R}}:=\left\{R \in \mathrm{SO}(3): V_{R}(R)<2 k_{R}\right\}$, $\operatorname{tr}\left(R_{e}(0) \neq-1\right.$ since $\operatorname{tr}\left(R_{e}\right)=-1 \Leftrightarrow V_{R}\left(R_{e}\right)=2 k_{R}$ and $V_{R}\left(R_{e}\right)$ is forced to decrease by continuity by virtue of (30) and $\gamma_{R}\left(R_{e}\right)$, in turn, is well-defined. Accordingly, for all initial conditions in $S_{V_{R}}$, the solutions of system (14) satisfy $V_{R}\left(R_{e}(t)\right)=V_{R}\left(R_{e}(0)\right) \exp \left(-\frac{k_{R}}{2} t\right) \quad \forall t \geq 0$. It is worth remarking that as subsets, $S_{R}$ and $S_{V_{R}}$ are equal, and therefore $S_{R}$ is positively invariant. Finally, since $V_{R}$ is quadratic, it can be easily shown that $\left\|R_{e}(t)\right\|_{\mathrm{SO}(3)} \leq \sqrt{2}\left\|R_{e}(0)\right\|_{\mathrm{SO}(3)} \exp \left(\frac{-k_{R}}{4} t\right) \quad \forall t \geq 0$, for all initial conditions starting in $S_{R}$. Finally, Property 1 is satisfied since for any $\ell \in(0,1)$ we have that $\left\{R \in \mathrm{SO}(3):\|R\|_{\mathrm{SO}(3)} \leq \ell\right\} \subseteq S_{R}$.

Remark 3: The stabilizer used in Proposition 2 is only one among several possible alternatives. By exploiting the angle axis parametrization $\mathbb{S}^{2} \times(-\pi, \pi) \ni(n, \theta) \mapsto R(n, \theta) \in \operatorname{SO}(3)$, it is readily seen that $\left\|\gamma_{R}\left(R_{e}(n, \theta)\right)\right\|=k_{R} \sqrt{\frac{1-\cos (\theta)}{2}} \leq k_{R}$, which reveals that the proposed solution is bounded for any $R_{e} \in S_{R}$ and that $\lim _{\theta \rightarrow \pm \pi}\left\|\gamma_{R}\right\|=k_{R}$. For large attitude errors, this is more aggressive than the standard selection $\gamma_{R}^{0}\left(R_{e}\right):=k_{R}$ skew $\left(R_{e}\right)^{\vee}$ which has a vanishing magnitude for $\theta \rightarrow \pm \pi$, since $\left\|\gamma_{R}^{0}\left(R_{e}(n, \theta)\right)\right\|=k_{R}|\sin (\theta)|$. For further details about this comparison see [13].

\section{B. Position stabilization}

A smooth version of the quasi time-optimal stabilizer for saturated double integrators, originally proposed in [14], and also used in [18], is employed as the position stabilizer in a decoupled form $\gamma_{p}\left(e_{x}, e_{v}\right)=$ $\left(\gamma_{p_{1}}\left(e_{x_{1}}, e_{v_{1}}\right), \gamma_{p_{2}}\left(e_{x_{2}}, e_{v_{2}}\right), \gamma_{p_{3}}\left(e_{x_{3}}, e_{v_{3}}\right)\right)$ :

$$
\gamma_{p_{i}}\left(e_{x_{i}}, e_{v_{i}}\right):=-\sigma_{M_{i}}\left(k_{x_{i}}\left(e_{x_{i}}+e_{v_{i}} \mu\left(\frac{\left|e_{v_{i}}\right|}{2 M_{i}}, \frac{k_{v_{i}}}{k_{x_{i}}}\right)\right)\right) \text {, }
$$

for $i \in\{1,2,3\}$, where $k_{x_{i}}, k_{v_{i}} \in \mathbb{R}_{>0}$ are scalar PD-like gains assigning the small-signal linear behavior, $\sigma_{M_{i}}(\cdot)$ denotes a 
continuously differentiable version of the saturation function satisfying $\sigma_{M_{i}}(s) s>0$ if $s \neq 0$, whose components $\sigma_{M_{i}}$ are bounded away from zero for large values of $s$ and are globally bounded by $\left|\sigma_{M_{i}}(s)\right| \leq M_{i}$ with $M_{i} \in \mathbb{R}_{>0}$ arbitrarily selected. Finally function $\mu(a, b):=\sqrt[n]{a^{n}+b^{n}}, a, b \in \mathbb{R}_{\leq 0}$, for some $n \geq$ 1 is a smooth approximation of the maximum of two nonnegative scalars. The next proposition establishes iISS from the attitude error input of the arising position closed-loop (12), (13), (31).

Proposition 3: Consider the selection $c\left(R_{e}\right):=1$ and the QTO position stabilizer (31). Then, Property 2 is satisfied.

Proof. The proof is based on [12, Lemma 1] establishing iISS if there exists a $C^{1}$, positive definite and radially unbounded function $V\left(e_{x}, e_{v}\right)$ and a class- $H_{I}$ function $\eta(\cdot)$ such that $\dot{V}\left(e_{x}, e_{v}\right) \leq \eta\left(\left\|R_{e}\right\|_{\mathrm{SO}(3)}\right)$. To this end, consider the following Lyapunov candidate for the unperturbed system, proposed in [18]:

$$
V_{p}\left(e_{x}, e_{v}\right):=\frac{1}{2} m \sum_{i=1}^{3} k_{x_{i}} e_{v_{i}}^{2}+\sum_{i=1}^{3} \int_{0}^{u_{i}} \sigma_{M_{i}}(s) d s,
$$

which is positive definite and radially unbounded from the stated sector properties of $\sigma_{M_{i}}$. Applying [15, Lem. 4.3] and using the structure of the first term of $V_{p}$, there exist class- $K_{\infty}$ functions $\alpha_{m}(\cdot), \alpha_{M}(\cdot)$ and a scalar $c_{v} \in \mathbb{R}_{>0}$ such that

$$
c_{v}\left\|e_{v}\right\|^{2}+\alpha_{m}\left(\left\|e_{x}\right\|\right) \leq V_{p}\left(e_{x}, e_{v}\right) \leq \alpha_{M}\left(\left\|e_{p}\right\|\right),
$$

where we denoted $e_{p}:=\left(e_{x}, e_{v}\right)$. By straightforward derivations we first obtain

$$
\begin{aligned}
& \frac{\partial V_{p}\left(e_{p}\right)}{\partial e_{x_{i}}}=-k_{x_{i}} \sigma_{M_{i}}\left(u_{i}\right) \\
& \frac{\partial V_{p}\left(e_{p}\right)}{\partial e_{v_{i}}}=m k_{x_{i}} e_{v_{i}}-k_{x_{i}} \sigma_{M_{i}}\left(u_{i}\right)\left(\mu_{i}+\frac{\partial \mu_{i}}{\partial e_{v_{i}}} e_{v_{i}}\right)
\end{aligned}
$$

where we used the shortcut notation $\mu_{i}:=\mu\left(\frac{\left|e_{v_{i}}\right|}{2 M_{i}}, \frac{k_{v_{i}}}{k_{x_{i}}}\right)$. Using $\mu_{i} \geq 0$ and $\frac{\partial \mu_{i}}{\partial e_{v_{i}}} e_{v_{i}} \geq 0$, the time derivative of $V_{p}$ along the unperturbed dynamics (12), (13), (31) (namely $\Delta R f_{d}=0$ ) satisfies

$$
\dot{V}_{p}\left(e_{x}, e_{v}\right)=-\frac{1}{m} \sum_{i=1}^{3} k_{x_{i}}\left(\mu_{i}+\frac{\partial \mu_{i}}{\partial e_{v_{i}}} e_{v_{i}}\right) \sigma_{M_{i}}^{2}\left(u_{i}\right) \leq 0 .
$$

The function $\mu_{i}$ is the $n$-norm of the vector $\left[\frac{\left|e_{v_{i}}\right|}{2 M_{i}} \frac{k_{v_{i}}}{k_{x_{i}}}\right]^{T}$, for which one has the bound $\mu_{i} \leq \frac{\left|e_{v_{i}}\right|}{2 M_{i}}+\frac{k_{v_{i}}}{k_{x_{i}}}$ and, by exploiting homogeneity, the same bound can be shown to hold also for $\frac{\partial \mu_{i}}{\partial e_{v_{i}}} e_{v_{i}}$. As a consequence, using the bounds and gradients in (33)-(35), for any $r>0$, we may establish the upper bound:

$$
\left\|e_{p}\right\| \geq r \Rightarrow \frac{\left\|\nabla_{e_{p}} V_{p}\left(e_{p}\right)\right\|}{V_{p}\left(e_{p}\right)} \leq \frac{c_{1}+c_{2}\left\|e_{v}\right\|}{c_{v}\left\|e_{v}\right\|^{2}+\alpha_{m}(r)} \leq c_{3},
$$

for some scalars $c_{1}, c_{2} \in \mathbb{R}_{>0}$, and $c_{3} \in \mathbb{R}_{>0}$ possibly growing unbounded as $r$ approaches zero. Inspired by (37), let us now define a $C^{1}$, positive definite and radially unbounded function

$$
V\left(e_{x}, e_{v}\right):=\left\{\begin{array}{cl}
\ln \left(V_{p}\left(e_{x}, e_{v}\right)\right) & \text { if } V_{p}\left(e_{x}, e_{v}\right)>\mathrm{e} \\
\frac{1}{\mathrm{e}} V_{p}\left(e_{x}, e_{v}\right) & \text { if } V_{p}\left(e_{x}, e_{v}\right) \leq \mathrm{e}
\end{array}\right.
$$

where e denotes Euler's number. Using (37) and the fact that the gradient of $V_{p}$ is bounded in any compact set $\left\|e_{p}\right\| \leq r$, we obtain $\left\|\nabla V\left(e_{p}\right)\right\| \leq c_{4}$ for some $c_{4} \in \mathbb{R}_{>0}$. Combining this bound with the complementary one in (37), inspecting the perturbed dynamics (12), (13), (31) and exploiting the unperturbed bound (36), we may write

$$
\dot{V}\left(e_{p}\right) \leq \max \left(c_{3}, c_{4}\right)\left\|\Delta R\left(R_{e}, e_{x}, e_{v}, R_{d}\right) f_{d}\left(e_{x}, e_{v}\right)\right\|,
$$

where we may use $\left\|\Delta R f_{d}\right\|$ as a shortcut for the right hand side interconnection term in (13). Consider now the chain of inequalities $\left\|\Delta R f_{d}\right\| \leq\|\Delta R\|\left\|f_{d}\right\| \leq\|\Delta R\|_{F}\left\|f_{d}\right\|$. Then, since $\left\|\Delta R\left(R_{e}\right)\right\|_{F}=\left\|R_{p}^{T} R_{e} R_{p}-I_{3}\right\|_{F}=\sqrt{6-2 \operatorname{tr}\left(R_{e}\right)} \leq$ $2 \sqrt{2}\left\|R_{e}\right\|_{\mathrm{SO}(3)}$ (by exploiting the definition of the Frobenius norm, the trace operator properties and the inequality $-1 \leq \operatorname{tr}(A) \leq 3$ for any $A \in \operatorname{SO}(3)$ [7, eqn. (86)]) and since $\left\|f_{d}\right\|=\left\|\gamma_{p}\left(e_{x}, e_{v}\right)+m_{g} e_{3}\right\| \leq \sum_{i=1}^{3} M_{i}+m g \leq T_{M}$ (by selecting $\sum_{i=1}^{3} M_{i} \leq T_{M}-m g$ as per (28)), one gets:

$$
\left\|\Delta R f_{d}\right\| \leq 2 \sqrt{2} T_{M}\left\|R_{e}\right\|_{\mathrm{SO}(3)} .
$$

Finally, combining bounds (39), (40), we get $\dot{V}\left(e_{p}\right) \leq 2 \sqrt{2} \max \left(c_{3}, c_{4}\right) T_{M}\left\|R_{e}\right\|_{\mathrm{SO}(3)}:=\eta\left(\left\|R_{e}\right\|_{\mathrm{SO}(3)}\right)$, where function $\eta(\cdot)$ is clearly of class- $H_{I}$, and we can apply [12, Lemma 1] to conclude that the $\left(e_{x}, e_{v}\right)$-subsystem is iISS with respect to input $R_{e}$ and that the QTO stabilizer (31) satisfies Property 2.

Remark 4: The selection $c\left(R_{e}\right):=1$ corresponds to the choice adopted by most hierarchical strategies [4], [5]. Different solutions can be envisaged to improve performance, e.g., $c\left(R_{e}\right):=\frac{\left.\bar{c}-\left(1-e_{3}^{T} R_{e} e_{3}\right)\right)}{\bar{c}}, \bar{c}>2$, which guarantees reduced position overshoot for large initial attitude errors (see Remark 1 and [6, Section VI-C]). With this choice, it can be shown that the proof of Proposition 3 still works.

\section{Simulation Results}

We present a simulation example to assess the performance of the proposed control law (10)-(11) when the attitude and position stabilizers are selected as in Propositions 2 and 3. We also compare the performance obtained with a nested saturations-based stabilizer, which is one of the most common solutions in the recent literature [5], [6], [4] to globally stabilize the position dynamics of UAVs. To better highlight the differences, we refer to ideal conditions in which the UAV is described by the control model (4)-(6). The considered UAV has mass $m=1 \mathrm{~kg}$ and can deliver a maximum thrust of $T_{M}=40 \mathrm{~N}$. The gain of the attitude stabilizer $\gamma_{R}(\cdot)$ used in Proposition 2 is selected as $k_{R}=40$. For the QTO stabilizer (31), the gains can be freely chosen to have a desired behavior in unsaturated conditions and are set to $k_{v_{i}}=9, k_{x_{i}}=\frac{k_{v_{i}}^{2}}{4}$ (critical damping). The saturation levels are set to $M_{i}=9$ for $i \in\{1,2,3\}$ so as to ensure the validity of (28). The nested saturations stabilizer used for comparison is the one employed in [5]:

$$
\gamma_{p}\left(e_{x}, e_{v}\right):=-\lambda_{2} \sigma_{1}\left(\frac{k_{2}}{\lambda_{2}}\left(e_{v}+\lambda_{1} \sigma_{1}\left(\frac{k_{1}}{\lambda_{1}} e_{x}\right)\right)\right)
$$

where the gains are set to $k_{1}=0.06, k_{2}=9$ and the saturation levels to $\lambda_{1}=5, \lambda_{2}=9$. While we have used the same outer saturation level $\lambda_{2} \equiv M_{i}$ and damping gain $k_{2} \equiv k_{v_{i}}$ of 
the QTO stabilizer, we had to follow the guidelines of [5, Prop. 1] in selecting $k_{1}$ and $\lambda_{1}$, to guarantee the small-signal ISS property. Different selections of the control parameters could be considered but the constraints among them [5, eq. (26)] ultimately pose an intrinsic limitation to the achievable performance possibly caused by the strong ISS robustness property. As already mentioned, this is a common issue shared by nested saturations-based stabilizers [10].

The initial state of the UAV is $x(0)=(4,4,4) \mathrm{m}$, $v(0)=(10,0,0) \mathrm{m} / \mathrm{s}$ and $R(0)=I_{3}+\sin \left(179.9^{\circ}\right) \hat{e}_{1}+(1-$ $\left.\cos \left(179.9^{\circ}\right) \hat{e}_{1}^{2}\right)$, which corresponds to an upside-down configuration with a significantly misplaced position with respect to the desired hovering position $x_{d}(0)=(0,0,1) \mathrm{m}, R_{d}=I_{3}$. The attitude tracking performance is illustrated in Figure 2 (top) for both controllers. Since the same control law is used for attitude stabilization, the same attitude transient is achieved. On the contrary, the position errors are characterized by a quite different behavior. The aggressive nature of the QTO stabilizer results in an impressively faster response (see Figure 2 (bottom)). This desirable aggressive behavior is confirmed by inspecting Figure 3, where the thrust and the magnitude of the commanded angular velocity are depicted.
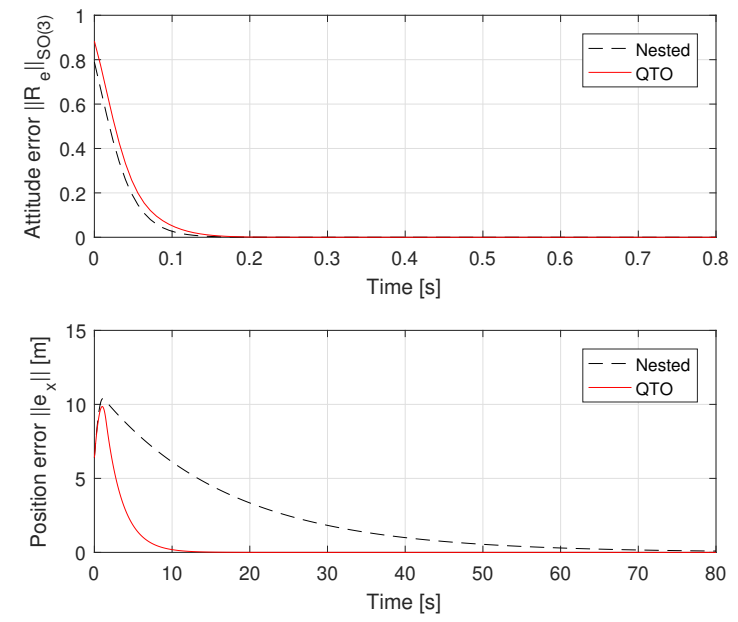

Figure 2. Stabilization errors: attitude $\left\|R_{e}\right\|_{\mathrm{SO}(3)}$ (top) and position $\left\|e_{x}\right\|$.

\section{CONCLUSIONS}

The stabilization problem for vectored-thrust UAVs has been addressed using an inner-outer loop paradigm and integral ISS tools to analyze the arising pseudo cascade. This allows one to employ stabilizers with superior performance with respect to existing strategies while guaranteeing the same basin of attraction. Our stabilization results can be extended to trajectory tracking following the approach in [6], which should allow tracking in cluttered environments via suitable planners.

\section{REFERENCES}

[1] M.-D. Hua, T. Hamel, P. Morin, and C. Samson, "Introduction to feedback control of underactuated VTOL vehicles: A review of basic control design ideas and principles," IEEE Control Systems, vol. 33, no. 1 , pp. $61-75,2013$.
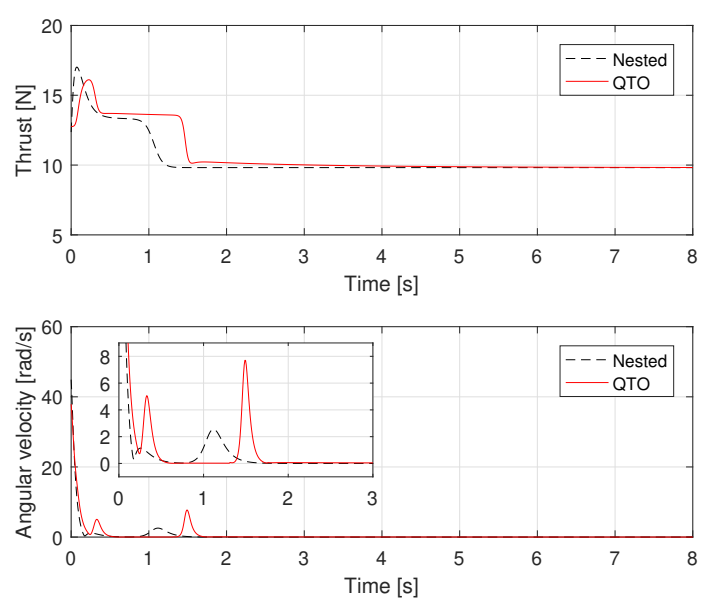

Figure 3. Control inputs: thrust $T_{c}$ (top) and angular velocity $\left\|\omega_{c}\right\|$.

[2] E. Frazzoli, M. Dehleh, and E. Feron, "Trajectory tracking control design for autonomous helicopters using a backstepping algorithm," in Proceedings of the American control conference, vol. 6, 2000, pp. 41024107.

[3] T. Hamel, R. Mahony, R. Lozano, and J. Ostrowski, "Dynamic modelling and configuration stabilization for an x4-flyer." IFAC Proceedings Volumes, vol. 35, no. 1, pp. 217-222, 2002.

[4] A. Roza and M. Maggiore, "A class of position controllers for underactuated VTOL vehicles," IEEE Transactions on Automatic Control, vol. 59 no. 9, pp. 2580-2585, 2014.

[5] R. Naldi, M. Furci, R. G. Sanfelice, and L. Marconi, "Robust global trajectory tracking for underactuated VTOL aerial vehicles using innerouter loop control paradigms," IEEE Transactions on Automatic Control, vol. 62, no. 1, pp. 97-112, 2017.

[6] D. Invernizzi, M. Lovera, and L. Zaccarian, "Geometric trajectory tracking with attitude planner for vectored-thrust VTOL UAVs," in American Control Conference, Milwaukee (WI), USA, Jul. 2018.

[7] — "Dynamic attitude planning for trajectory tracking in underactuated UAVs," arXiv:1810.04494, 2018.

[8] M. Maggiore, M. Sassano, and L. Zaccarian, "Reduction theorems for hybrid dynamical systems," IEEE Transactions on Automatic Control, 2019, to appear.

[9] A. Chaillet, D. Angeli, and H. Ito, "Combining iISS and ISS with respect to small inputs: the strong iISS property," IEEE Transactions on Automatic Control, vol. 59, no. 9, pp. 2518-2524, Sep. 2014.

[10] N. Marchand and A. Hably, "Global stabilization of multiple integrators with bounded controls," Automatica, vol. 41, no. 12, pp. 2147-2152, 2005.

[11] E. D. Sontag, "Comments on integral variants of ISS," Systems \& Control Letters, vol. 34, no. 1, pp. 93-100, 1998.

[12] M. Arcak, D. Angeli, and E. Sontag, "A unifying integral ISS framework for stability of nonlinear cascades," SIAM Journal on Control and Optimization, vol. 40, no. 6, pp. 1888-1904, 2002.

[13] T. Lee, "Exponential stability of an attitude tracking control system on so (3) for large-angle rotational maneuvers," Systems \& Control Letters, vol. 61 , no. 1, pp. 231-237, 2012.

[14] F. Forni, S. Galeani, and L. Zaccarian, "A family of global stabilizers for quasi-optimal control of planar linear saturated systems," IEEE Transactions on Automatic Control, vol. 55, no. 5, pp. 1175-1180, May 2010.

[15] H. K. Khalil, Nonlinear systems, 3rd Edition. Prentice-Hall, 2002.

[16] D. E. Koditschek, "The Application of Total Energy as a Lyapunov Function for Mechanical Control Systems," J. E. Marsden, P. S. Krishnaprasad and J. C. Simo (Eds) Dynamics and Control of Multi Body Systems, vol. 97, pp. 131-157, February 1989.

[17] C. G. Mayhew, R. G. Sanfelice, and A. R. Teel, "Quaternion-based hybrid control for robust global attitude tracking," IEEE Transactions on Automatic Control, vol. 56, no. 11, pp. 2555-2566, Nov 2011.

[18] M. Andreetto, D. Fontanelli, and L. Zaccarian, "Quasi time-optimal hybrid trajectory tracking of an n-dimensional saturated double integrator,' in Proceedings of IEEE Conference on Control Applications (CCA), 2016, pp. 550-555. 